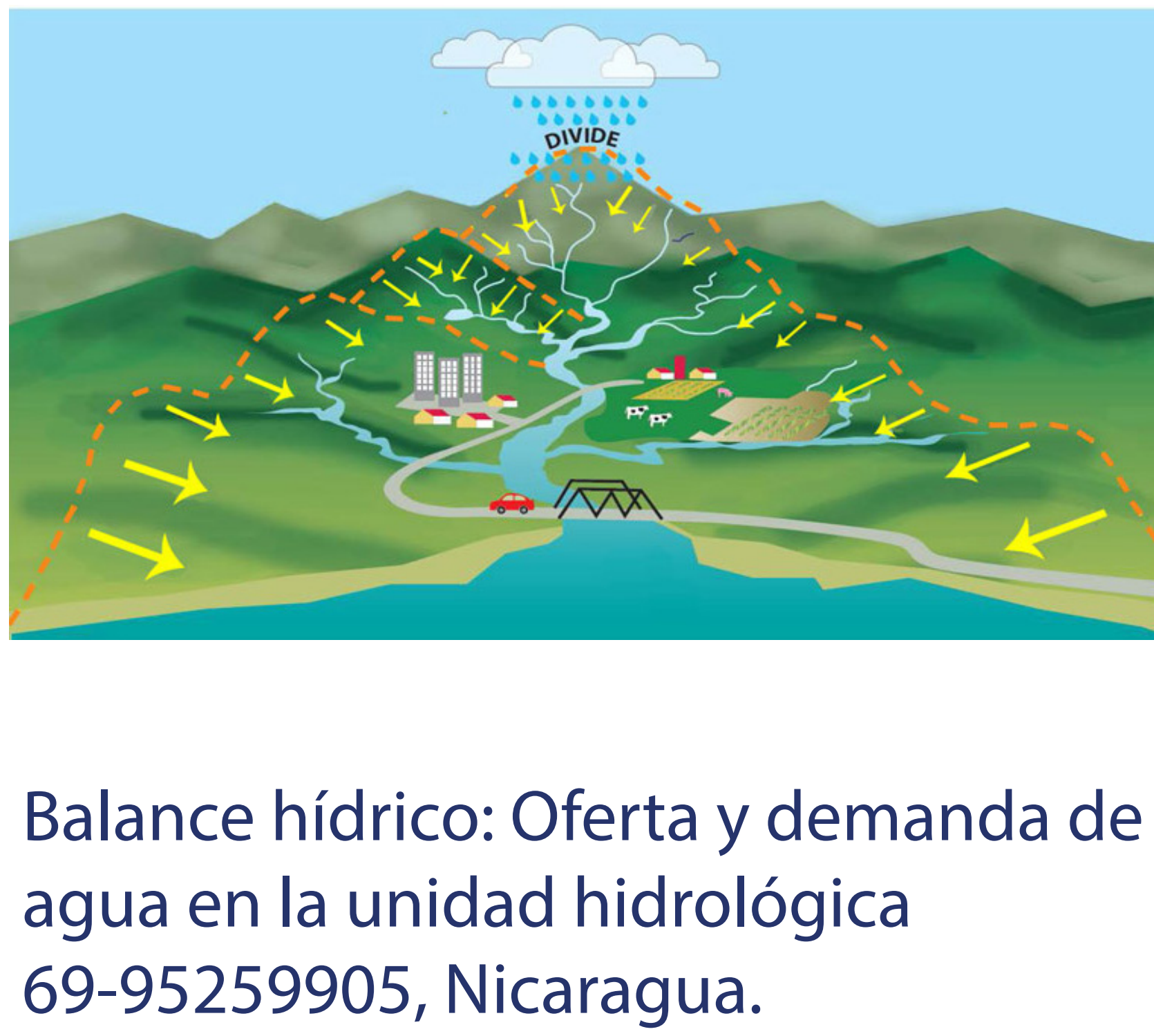

Norman Josué García García

Licenciado en Geografía, UNAN MANAGUA, Departamento de Geografía

https://orcid.org/0000-0002-5317-8908

normam1618@gmail.com

Nereyda Fabiola Salinas.

Licenciada en Geografía, UNAN MANAGUA, Departamento de Geografía

https://orcid.org/0000-0002-8114-5943

nereydasalinas293@gmail.com

Lisseth Carolina Blandón Chavarría.

Master en Ciencias del agua con énfasis en calidad. UNAN MANAGUA, Departamento de Geografía.

https://orcid.org/0000-0003-4125-0332

lizzblandon@gmail.com 


\title{
Balance hídrico: Oferta y demanda de agua en la unidad hidrológica 69-95259905, Nicaragua.
}

\author{
Water balance: Water supply and demand in the \\ hydrological unit 69-95259905, Nicaragua
}

\section{RESUMEN}

La determinación del balance hídrico y su cuantificación rigurosa es uno de los elementos esenciales para determinar la oferta y demanda de agua en cuencas hidrográficas. El presente estudio se realizó en la unidad hidrológica 69-95259905, considerando que no se tiene estudio de oferta y demanda de agua. Se empleo el método de recarga potencial de acuíferos mediante un balance hídrico de suelos (Gunther Schosinsky N); los resultados mostraron que actualmente la oferta de agua en la cuenca supera la demanda para consumo humano y actividades económicas.

\section{ABSTRACT}

The establishment of the water balance and its rigorous quantification is one of the essential elements to determine the supply and demand of water in hydrographic basins. The present study was carried out in the hydrological unit 69-95259905, considering that there is no study of water supply and demand. The method of potential recharge of aquifers was used by means of a soil water balance (Gunther Schosinsky $\mathrm{N}$ ); The results showed that currently the water supply in the basin

\section{PALABRAS CLAVE}

Cuenca, suministro de agua. demanda de agua, valance hidrico.

\section{KEYWORDS}

\author{
Basin, Water supply, Water demand, Water \\ balance
} exceeds the demand for human consumption and economic

\section{Introducción}

Nicaragua posee abundantes recursos hídricos tanto superficiales como subterráneos y la mejor manera de gestionar los recursos hídricos debe ser desde el enfoque de cuenca hidrográfica, como unidad de gestión administrativa, de ello depende el buen desarrollo de las diferentes actividades que giran en torno a la conservación y administración del agua (ANA, 2017).

De cara a esta mención en el país se han realizado importantes proyectos como el de 1960-1972, PHCA (Proyecto Hidrometeorológico Centroamericano) que da como resultado la determinación en el país de 21 cuencas y 218 subcuencas hidrográficas, con visión hacia el aprovechamiento hidroeléctrico; es en el año 2014 que se presenta una nueva delimitación de cuencas bajo la metodología Pfafstetter (1989); la que muestra las diferentes cuencas hidrográficas y la respectiva codificación, esto marca una nueva etapa en la búsqueda de una gestión integral de los recursos hídricos del país.

Sin embargo, el gestionar los recursos hídricos no es tarea fácil, en la actualidad en muchas comunidades del país existe una demanda creciente del recurso y el determinar la disponibilidad se debe hacer más detallada, por ello la presente investigación es un estudio determino la disponibilidad hídrica a un nivel micro con el enfoque y concepto de cuenca hidrográfica, este aporte se suma a los esfuerzos de las pertinentes instituciones que trabajan por la gestión integral de los recursos hídricos, así como para mejorar las condiciones de acceso al recurso de los comunitarios en la cuenca. 
La cuenca en estudio pertenece a la subcuenca Laguna de Masaya, el 99.03\% del territorio está ocupada por área urbana del municipio de Masaya y el 0,97\% por el municipio de Catarina, INETER (2006), en ella se encuentran siete comunidades: El Túnel, La Poma, Pacayita, Pacaya, Nandayure y El Mojón.

Se determinó la oferta y demanda de agua en la cuenca, a partir del método de recarga potencial de acuíferos mediante un balance hídrico de suelos (Gunther Schosinsky N), además se utilizaron datos de población, datos de consumo histórico-actual y con ello se determinó la demanda del recurso y la disponibilidad que existe para solventar el consumo humano y actividades económicas de los habitantes.

Es importante mencionar que el suministro se agua en algunas comunidades en la cuenca no es estable, lo que trae como consecuencias que la población cave huecos cerca de la tubería madre en los camino de las comunidades y extraigan el agua, puesto que es poca la cantidad de agua suministrada; en otra ocasiones las familias se ven obligadas a comprar el agua por barriles. Durante esta investigación se busca determinar si el suministro o demanda insatisfecha en el área está vinculado con: flujos insuficientes al entorno natural, insuficiente infraestructura, sobreexplotación del recurso, bajos ingresos económicos de población para pagar el servicio $y$, conflictos por uso de agua.

\section{Material y Método}

\section{Tipo de investigación}

La investigación es de tipo descriptiva puesto que caracteriza un hecho, establece explicaciones y correlaciones entre variables físicas, económicos y sociales en el área en estudio.

\section{Fuentes y técnicas de recopilación de datos.}

Se recopilaron en formato de Excel, datos de precipitación, temperatura máxima, media y mínima, proporcionados por INETER, se retomó la metodología del balance de suelo de (Schosinsky G. , 2006). A demás de ello se retoma información de suelo, pruebas de infiltración y, así como parámetros hidráulicos de la porción del acuífero de la cuenca.

\section{Disponibilidad hídrica}

Para llevar a cabo la realización del balance hídrico se recopilaron, depuraron y analizaron una serie de datos o variables, en el siguiente acápite se detalla el método de corrección o aplicación si fuese necesario.

\section{Precipitación}

Se realizóla sistematización de la información delas estaciones pluviométricas meteorológicas comprendidas en periodo de 35 años (1983-2017), las estaciones que se consideraron fueron las adyacentes a la cuenca. Los datos de precipitación fueron proporcionados por INETER, provenientes del área de climatología.

Se realizóla sistematización dela información delas estaciones pluviométricas meteorológicas comprendidas en periodo de 35 años (1983-2017), las estaciones que se consideraron fueron las adyacentes a la cuenca. Los datos de precipitación fueron proporcionados por INETER, provenientes del área de climatología.

\begin{tabular}{|c|c|c|c|c|c|c|}
\hline Código & Estación & Tipo & Período & X Coord. & Y coord. & $\begin{array}{c}\text { Precipitación Anual } \\
\text { mm. }\end{array}$ \\
\hline 69115 & Masaya & HM & 35 años & 587439 & 1324500 & 113.81 \\
\hline 69129 & $\begin{array}{c}\text { Campos } \\
\text { Azules }\end{array}$ & HM & 35 años & 592597 & 1315606 & 225.91 \\
\hline
\end{tabular}

Tabla 1: Estaciones pluviometricas metereológicas aledañas a la cuenca. Fuente: INETER. 
Estimación de la distribución espacial de la precipitación (método promedio aritmético).

Para la estimación de la distribución espacial de la precipitación en la cuenca de estudio, se aplicó como primer paso el coeficiente de correlación ya que solo se cuenta con dos estaciones meteorológicas, pero con mucha influencia y cercanía a la cuenca, posterior se realizó el promedio aritmético que consiste en promediar los valores de precipitación registrados en las estaciones de la cuenca hidrográfica.

\section{Temperatura}

Para determinar la temperatura se tomaron los datos registrados mensualmente de las estaciones meteorológicas Masaya y Campos Azules, su análisis se realizó en Excel para determinar la temperatura absoluta de la mínima, media y máxima.

\section{Evapotranspiración Potencial.}

La evapotranspiración potencial (ETP) se calculó a través del método de Hargreaves, utilizando datos de temperatura de la estación Campos Azules y estación Masaya. La fórmula empleada es la siguiente:

$E T P=0.0023 *(T m e d+17.78) * R o$

$*(\operatorname{Tmax}-\operatorname{Tmin})^{\wedge} 2$

Tmed: Temperatura media

Ro: Radiación solar extraterrestre

Tmax: Temperatura máxima absoluta

Tmin: Temperatura mínima absoluta

\section{Los componentes son de BHS:}

a. Precipitación media mensual ( $\mathrm{mm}$ )

b. Coeficiente de infiltración $(C f)=0.88 C, y C=K p+K v+K f c$. (Schosinsky \& Losilla, 2000).

c. Capacidad de Infiltración ( $F c$ ): Tabla de referencia

d. Capacidad de campo de suelo: Tablas de referencia.

e. Punto de marchitez: Tabla de referencia.

\section{Determinación de la recarga potencial}

Se determinó la recarga por medio del Balance Hídrico de suelo propuesto por (Schosinsky, 2006). Se obtuvo la recarga potencial de aguas subterráneas. Los principales componentes del balance hídrico de suelo son: Precipitación media del área en estudio (P), Coeficiente de infiltración (Cf), Precipitación que se infiltra (I), Precipitación que se escurre (Esc), Evapotranspiración potencial (ETP), humedad del suelo inicial (Hsi), agua disponible (Agd), humedad del suelo final (Hsf), déficit de capacidad de campo(DCC), Evapotranspiración real (ETR), recarga potencial del acuífero (Rp). 
Determinación del balance hídrico subterráneo.

Para determinar la disponibilidad de agua en la cuenca se utilizó la fórmula del balance subterráneo, se basa en el principio de conservación de masa y establece que para cualquier volumen arbitrario y durante cualquier periodo de tiempo, la diferencia entre las entradas y salidas estará condicionada por la variación del volumen de agua almacenada.

Ecuación: $(R P+Q c+R r)-(B+Q r+Q p+E s u b+Q a)=\Delta S$

\section{Entrada:}

Rp: Recarga directa por precipitación al terreno, tomada del Balance hídrico de suelo.

$Q \boldsymbol{Q}$ : Infiltración de corrientes superficiales si existiesen en la cuenca de estudio.

Rr: Recarga por retorno

\section{Salidas:}

B: Bombeo, (volumen de extracción) tomado de información recopilada de pozos cercanos al área de estudio.

Qr: Salidas a través de ríos.



\section{Resultado y discusón}

\section{Oferta y demanda del recurso hídrico en la cuenca}

Las cuencas hidrográficas son sistemas hidrológicos que reciben y procesan entradas de agua y producen salidas de ella. Por tanto, las cuencas son susceptibles a ser sometidas a un análisis de balance hídrico cuando se cuenta con información suficiente y buena para ello (Silva León, 2004). Precisamente en la cuenca en estudio no se tiene en su totalidad los datos requeridos; por lo cual debe considerarse este Balance Hídrico como un resultado preliminar. 


\section{Precipitación}

Para determinar la precipitación areal mensual de la cuenca se aplicó el método de la media aritmética con las estaciones Masaya (69115) y Campos Azules (69129), a través de coeficiente de correlación se comprobó si existe una estrecha relación entre los datos de las estaciones dando como resultado $0.98 \%$ de confianza.

En la cuenca se presentan dos periodos bien definidos; uno lluvioso que va desde mayo y finaliza en octubre, el cual se puede subdividir en dos sub periodos; el primero inicia en mayo y finaliza en julio, el segundo de agosto a octubre; en este periodo se acumulan $1293.49 \mathrm{~mm}$ anuales que equivale al $91.10 \%$ del total de lluvia que cae sobre la cuenca, así mismo un periodo relativamente seco que se extiende de noviembre a abril con un acumulado de $126.36 \mathrm{~mm}$ correspondiente al 8.90\%, en otras palabras el régimen de precipitación incidente en la dinámica hidrológica está marcado por las precipitaciones del periodo lluvioso.

Distribución de la precipitación durante el periodo lluvioso.

En los primeros tres meses del periodo (mayo - julio) se acumulan $579.59 \mathrm{~mm}$ equivalente al $44.81 \%$ del periodo y en el segundo trimestre de agosto a octubre $713.89 \mathrm{~mm}$ equivalente al $55.19 \%$ con respecto al acumulado anual del periodo lluvioso.

De acuerdo al gráfico 2, a inicios del periodo lluvioso se observan precipitaciones de $194.32 \mathrm{~mm}$ en mayo y $221.93 \mathrm{~mm}$ en junio. En julio con 163.34 y agosto con $178.63 \mathrm{~mm}$ se presenta un descenso en la precipitación, debido al establecimiento del periodo canicular, los máximos mensuales de este periodo lluvioso se presentan en septiembre con $262.22 \mathrm{~mm}$ seguido de octubre con 273.04, debido al acercamiento de sistemas sinópticos propios de la temporada.

\section{Distribución de la precipitación durante el periodo seco}

El periodo seco de acuerdo al (grafico 2) inicia en el mes de noviembre y finaliza en abril, el mes más húmedo es noviembre con $73.92 \mathrm{~mm}$ y los meses más secos son febrero y marzo con $3.53 \mathrm{~mm}$ y $5.57 \mathrm{~mm}$. Durante todo el periodo seco se acumula un total de $126.36 \mathrm{~mm}$ anuales.



Gráfico 2: Comportamiento de la precipitación mensual de la cuenca 69-95259905 Fuente: INETER 


\section{Temperatura}

El grafico 3, representa el régimen térmico de la máxima, media y mínima, los valores de media mensual indican un comportamiento estable, en relación con los meses del periodo lluvioso de mayo a octubre, la temperatura media es de $25.25^{\circ} \mathrm{C}$, se observa que los meses con mayor temperatura son mayo con $26.72^{\circ} \mathrm{C}$ y agosto con $25.26^{\circ} \mathrm{C}$, descendiendo paulatinamente hasta el mes de octubre con $24.80^{\circ} \mathrm{C}$. Para el periodo seco del año los meses con las medias más altas son los meses de abril y marzo con 27.09 y 25.92 ${ }^{\circ} \mathrm{C}$ respectivamente, descendiendo en los meses de noviembre y diciembre debido a las masas de frente frio provenientes de las latitudes norte y que coinciden con la estación de invierno en el hemisferio, para el periodo seco se tiene una media de $25.49^{\circ} \mathrm{C}$.



Gráfico 3: Temperatura media mensual de la cuenca 69-95259905
Fuente: INETER

Los valores máximos de evapotranspiración se presentan en el periodo seco en los meses de febrero (119.31) y marzo (151.49), para todo el periodo seco se tiene un acumulado de $538.05 \mathrm{~mm}$, que corresponde al $54.10 \%$ de evapotranspiración total, esto se relaciona con el comportamiento de la temperatura, que coincide con las mayores para estos meses.

Para los meses del periodo lluvioso se mantiene una evapotranspiración estable entre los rangos de 56.13 y los $76.88 \mathrm{~mm}$, exceptuando el mes de octubre con $115.40 \mathrm{~mm}$, en este periodo se tiene un acumulado de 454.89 que equivale al $45.81 \%$ de precipitación que se evapotranspira.




Entrada del balance hidrico.

\section{Recarga directa.}

Se obtuvieron valores de infiltración entre 43.20 y 80,81 mm/d, con un promedio para toda la cuenca de $62.035 \mathrm{~mm} / \mathrm{d}$, características de suelos con textura franco. Las condiciones de recarga en la cuenca se deben al ambiente geológico de la formación Las Sierras, los estratos volcánicos de los bloques de Ticuantepe y pómez de apoyo son materiales permeables. Aplicando el método del balance hídrico de suelos (Schosinsky G. , 2006) se obtuvieron los valores de recarga potencial a continuación se detalla:

El balance indica una recarga de 2.96 mmca (millones de metros cúbicos anuales). En el grafico 5 se observa que, en los meses de enero, febrero, marzo abril las precipitaciones en la cuenca son mínimas y la ETP es mayor correspondiendo con los meses de mayor temperatura, por lo que para estos meses no se tiene recarga hídrica significativa, para el mes de mayo a pesar de que se inicia el periodo lluvioso con $194.32 \mathrm{~mm}$ el agua se pierde por evapotranspiración, escorrentía, así en los espacios porosos del suelo y que utilizan las diferentes coberturas para su desarrollo para dar inicio a la capacidad de campo.

\begin{tabular}{|c|c|c|c|c|}
\hline $\begin{array}{c}\text { Tipo de } \\
\text { suelo }\end{array}$ & $\begin{array}{c}\text { Coeficiente de } \\
\text { infiltración( } \mathrm{mm} / \text { día) }\end{array}$ & $\begin{array}{l}\text { Recarga } \\
\text { potencial }(\mathrm{mm})\end{array}$ & $\begin{array}{c}\text { Área de la cuenca } \\
\text { en } \mathrm{km}^{2}\end{array}$ & $\begin{array}{c}\text { Recarga potencial } \\
\text { (MMCA) }\end{array}$ \\
\hline Mollisols & 62.035 & 233.03 & 12.7 & 2.96 \\
\hline
\end{tabular}

Tabla 2: Recarga de acuiferos

Fuente: INETER

En los meses de junio a octubre las precipitaciones son más abundantes, permitiendo una recarga al acuífero, para los meses de noviembre y diciembre las precipitaciones disminuyen considerablemente perdiéndose el agua por evapotranspiración. En la cuenca de $1419.89 \mathrm{~mm}$ anuales que representa el $100 \%, 69.93 \%$ se pierde por evapotranspiración, $29.81 \%$ por escorrentía y el $16.41 \%$ se recarga al acuífero.

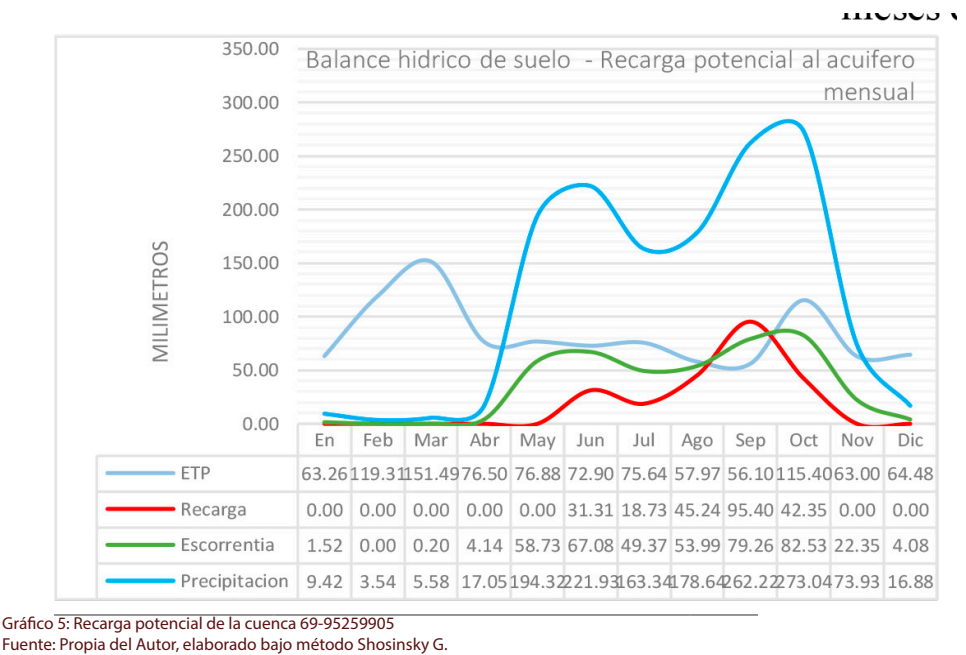




\section{Infiltración de corrientes superficiales}

La infiltración de corrientes superficiales se toma cuando en la cuenca se tienen ríos que aportan a la infiltración en la cuenca.

\section{Recarga por retorno de extracciones de pozos en la cuenca.}

Se determinó la extracción de los pozos que se encuentran en la cuenca y que son de suministro de agua potable para consumo humano, se estimó una extracción de $0.67 \mathrm{mmca}$, la recarga por retorno generada por bombeo se consideró en base a la“Norma para el Diseño de Sistemas de Abastecimiento y Potabilización de las Aguas" (NTON 09003 - 99), se toma el 20\% del sistema de agua potable, considerándolo como fuga o desperdicio del sistema, por lo cual constituye 0.13 mmca.

\section{Salidas del sistema (Balance hídrico) \\ Escurrimiento subterráneo}

La determinación del flujo de escurrimiento subterráneo fue calculada mediante la Ley de Esub=T*i*L, con datos de piezometría y Transmisividad proporcionados por INETER. El agua escurre con dirección al noroeste, desde las zonas más altas de las lomas ubicadas al suroeste de la cuenca, esto indica que el agua que sale de la formación acuífera por escurrimiento toma dirección a Laguna de Masaya.

El cálculo del gradiente hidráulico:

i: $325-115=210 \mathrm{~m} / 7286=0.0288$

Aplicando la Ley de Darcy:

Esub $=180 \mathrm{~m} 3 / \mathrm{d}^{*} 7286 \mathrm{~m}^{*} 365$ días*0.028 $^{*} 0.02$

Esub $=13,786,277.76=13.4 \mathrm{mmca}$

El escurrimiento subterráneo corresponde a 13.4 millones de metros cúbicos anualmente.

\begin{tabular}{|c|c|c|l|c|c|}
\hline $\begin{array}{c}\text { Área de } \\
\text { estudio }\end{array}$ & $\begin{array}{c}\text { Número } \\
\text { de } \\
\text { sección }\end{array}$ & $\begin{array}{c}\text { Transmisividad } \\
\text { (m3/día) }\end{array}$ & $\begin{array}{c}\text { Gradiente } \\
\text { hidráulico }\end{array}$ & $\begin{array}{c}\text { Longitud } \\
\text { de la } \\
\text { sección }\end{array}$ & $\begin{array}{c}\text { Esub } \\
\text { (mmca) }\end{array}$ \\
\hline $\begin{array}{c}\text { Cuenca 69- } \\
952509\end{array}$ & 1.00 & 180 & 0.0288 & 7286 & 13.4 \\
\hline
\end{tabular}

Tabla 3: Escurrimiento subterráneo de la cuenca 69-95259905

Fuente: INETER

Salida a través de ríos

Para obtener el dato se realizan campañas de aforo en los caudales visibles de los ríos en la zona de estudio, en este caso no se cuenta con ríos permanentes en la zona.

\section{Salida a través de otros acuíferos}

Para efectos de este estudio el medio del acuífero de la cuenca se ha considerar cerrada desde es el punto de vista hidrográfico e hidráulico. 


\section{Extracción por bombeo}

Para cuantificar la extracción de agua subterránea se identificaron en el área 3 pozos perforados, un pozo administrado por la empresa ENACAL y dos pozos privados, todos para uso de consumo humano, se obtuvo información de volumen de agua extraída por periodos semanales, a partir de este dato se proyectó la extracción por bombeo anualmente, los resultados se detallan en la siguiente tabla.

\begin{tabular}{|c|c|c|c|}
\hline $\begin{array}{c}\text { Pozo } \\
\text { perforado }\end{array}$ & $\begin{array}{c}\text { Coordenada } \\
\text { X }\end{array}$ & $\begin{array}{c}\text { Coordenada } \\
\text { Y }\end{array}$ & $\begin{array}{c}\text { Extracción } \\
\text { anual }\end{array}$ \\
\hline $\begin{array}{c}\text { Nueva } \\
\text { Esperanza }\end{array}$ & 600579 & 1322912 & $0.15 \mathrm{mmca}$ \\
\hline Masinfa & 600302 & 1222526 & $0.30 \mathrm{mmca}$ \\
\hline $\begin{array}{l}\text { ENACAL- } \\
\text { Sabogales }\end{array}$ & 599829 & 1322746 & $0.22 \mathrm{mmca}$ \\
\hline \multicolumn{4}{|l|}{} \\
\hline
\end{tabular}

\section{Percolación profunda}

El término de la percolación profunda es considerado aquel flujo que por efecto de gravedad desciende hacia las capas más profundas que son menos permeables o impermeables (Peña, 2005); para la zona de estudio no se tiene precisión del dato porque es necesario conocer la transición entre las capas. Por tanto, por estudios análogos a este se considera una pérdida del $5 \%$ de la recarga potencial, debido al tipo de roca y a la composición lito estratigráfica de la formación La Sierra, que varía desde arenas hasta tobas y cenizas volcánicas. El valor a introducir en la ecuación es 0.15 mmca.

\section{Consideración de recarga lateral al acuífero:}

En la mayoría de los casos la cuenca es razonablemente congruente con la cuenca hidrogeológica (Walton, 1970) citado por (Schosinsky G. , 2006), lo que quiere decir que la recarga o descarga se podrá calcular con la fórmula del escurrimiento subterráneo; en este caso la cuenca de estudio forma parte de una porción del acuífero Meseta de Carazo, por ende se considera una recarga lateral igual al escurrimiento subterráneo. El dato a utilizar en el balance es de 13.4 mmca.

\section{Balance hídrico subterráneo}

La variación del almacenamiento se referirá a la cuantificación de las reservas o déficit de agua en la cuenca de estudio, con el fin de estimar un equilibrio entre las entradas y salidas al sistema.

Como entrada al sistema se obtienen $16.49 \mathrm{mmca}$, la mayor parte del agua que entra a la cuenca es por infiltración de la precipitación, aunque notablemente está influida por la evapotranspiración en los meses más secos del año, haciendo que los regímenes de infiltración estén ligados a una adecuada cobertura y uso del suelo. 
Las salidas cuantificadas tanto por las extracciones, y características hidráulicas de la porción de acuífero contabilizan un total de $14.22 \mathrm{mmca}$, por lo cual se tiene un excedente disponible de $2.27 \mathrm{mmca}$ (Ver ilustración 1)

\begin{tabular}{|c|c|c|c|}
\hline ENTRADAS & MM3/anual & SALIDAS & $\mathrm{MM}^{3} /$ anual \\
\hline Recarga Potencial & 2.96 & Escurrimiento subterráneo & 13.4 \\
\hline Infiltración por corrientes superficiales & 0 & Salida a través de ríos & 0 \\
\hline Recarga por retorno de extracciones & 0.13 & Salida a través de otros acuíferos & $S D$ \\
\hline Recarga Lateral & 13.4 & Extracción por bombeo & 0.67 \\
\hline & & Percolación Profunda & 0.15 \\
\hline Total & 16.49 & Total & 14.22 \\
\hline
\end{tabular}

Tabla 5: Balance hídrico subterráneo de la cuenca 69-95259905

Fuente: INETER y datos propios de los autores.

Balance hídrico subterráneo

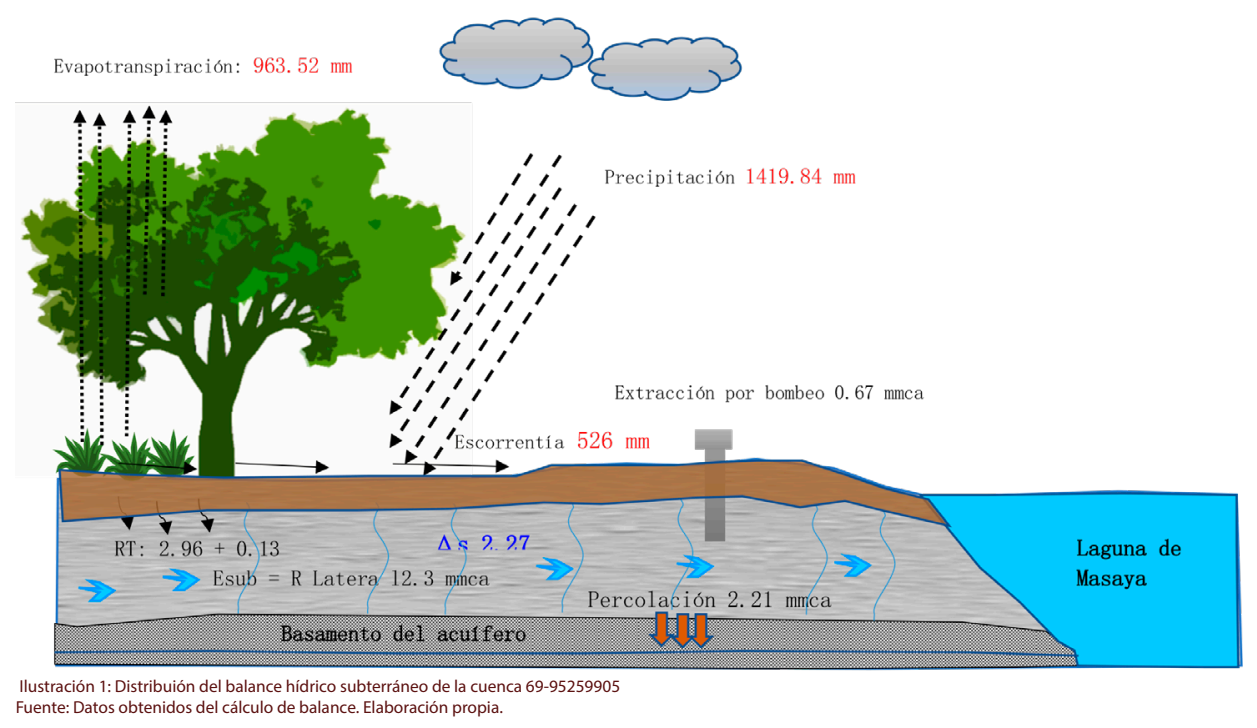

\section{Demanda}

Para conocer la demanda se recopiló información acerca de la población total que tiene problemas con la accesibilidad y estabilidad del servicio de agua potable y que se encuentran en la cuenca, a través del censo poblacional (INIDE, 2005), en donde se obtuvo el total de los habitantes por cada comunidad que abarca la cuenca; un insumo necesario fue el conocer el promedio de consumo por habitante, este dato se estimó por información proporcionada por los administradores de los pozos que se encuentran en la cuenca, el cual corresponde a $0.07 \mathrm{~m} 3$ diario, se proyectó al año 2020 con el dato de la tasa de crecimiento anual de(AMUDEMAS, 2009) que corresponde a $1.8 \%$. 
Con la población del año 2005 se tenía un consumo de $0.11 \mathrm{mmca}$, en cambio la proyección al año 2020 muestra $0.25 \mathrm{mmca}$, en este caso sigue habiendo agua disponible en la cuenca, ya que se cuenta con un total de $2.27 \mathrm{mmca}$.

\begin{tabular}{|c|c|c|c|c|}
\hline Comunidad & Población 2005 & Consumo anual 2005 & Población 2020 & Consumo anual 2020 \\
\hline Pacayita & 3214.00 & $82117.70 \mathrm{m3}$ & 4082.00 & $180612.95 \mathrm{m3}$ \\
\hline El Túnel & 1169.00 & $29867.95 \mathrm{m3}$ & 1485.00 & $65689.05 \mathrm{m3}$ \\
\hline Nandayure & 71.00 & $1814.05 \mathrm{m3}$ & 5658.00 & $3990.91 \mathrm{m3}$ \\
\hline Total & 4454.00 & $113799.70 \mathrm{m3}$ & & $0.25 \mathrm{mmca}$ \\
\hline
\end{tabular}

\section{Conclusiones}

De acuerdo a los resultados; la cuenca tiene agua disponible para satisfacer la demanda actual y futura; además de ello el actual desabastecimiento no esta vinculado a los flujos del entorno natural. Se deberá continuar con investigaciones que permitan establecer la causa actual del desabastecimiento en las comunidades.

La cuenca en cuanto a disponibilidad hídrica subterránea posee $2.27 \mathrm{mmca}$, la recarga hídrica está influenciada por los procesos de evapotranspiración y que van de acuerdo a la cobertura y uso del suelo de la cuenca, en el periodo seco los meses de mayor evapotranspiración son marzo y abril con un total de $270.80 \mathrm{~mm}$, lo que influye que para estos meses la recarga sea nula en el periodo lluvioso las precipitaciones aumentan por lo que porcentaje del agua caído en la cuenca llega al acuífero.

Los parámetros hidráulicos del acuífero indican una recarga lateral y escurrimiento subterráneo de 12.3 mmca, con dirección de flujo a la Laguna de Masaya.

Las extracciones de los pozos contabilizadas en $2.6 \mathrm{mmca}$ no exceden un volumen al de la recarga potencial, por lo que se llega a la conclusión que no hay sobreexplotación del recurso en la cuenca, sin embargo, debido a que es un balance de referencia se debe de cuantificar otras extracciones que existiesen en la cuenca.

\section{Agradecimientos}

A la universidad Nacional autónoma de Nicaragua, en especial al departamento de Geografía. Al instituto Nicaragüense de Estudios Territoriales en especial a: Ing. Gonzalo Bonilla, Ing. Wilmer Rodríguez, Ing. José Ariel Cruz, Ing. Gloria Pérez, Ing. Manuel Arreola, Ing. Erwin Rueda, Enoc Castillo, Marcial Peralta 


\section{Referencias Bobliográficas.}

- AMUDEMAS. (2009). Masaya en cifras. Obtenido de http://www.aecid.org.ni/wp-content/uploads/2014/04/ CARACTERIZACION-DEL-DEPARTAMENTO-DE-MASAYA-23-NOV-2009.pdf

- ANA. (agosto de 2017). Plan de Gestión Integrada de los Recursos Hídricos de la Cuenca 72 Entre Río Brito y Río Sapoá. Obtenido de Proatas: http://proatas.org.ni/media/flatpages/Plan_GIRH_72_2017_final_ impresi\%C3\%B3n.pdf

- Béjar, M. V. (2006). Hidrología Estadística. Cartago: Editorial Tecnológica de Cost Rica.

- INIDE. (2005). Censo poblacional 2005. Obtenido de https://www.inide.gob.ni/Estadisticas/ censoCEPOV2005

- Peña, E. (agosto de 2005). Disponiblidad y aprovechamiento sostenible del acuifero de Nandaime.

- Schosinsky, \& Losilla. (2000). Modelo analítico para determinar la infiltración con base en la lluvia mensual. Obtenido de https://revistas.ucr.ac.cr/index.php/geologica/article/download/8579/8102/

- Schosinsky, G. (2006). Cálculo de la recarga potencial de acuíferos mediante un balance hidríco de suelo. Obtenido de https://revistas.ucr.ac.cr/index.php/geologica/article/download/4223/4047

- Walton. (1970). Ground water resource evaluation.

Norman Josué García García

Licenciado en Geografía, UNAN MANAGUA, Departamento de Geografía https://orcid.org/0000-0002-5317-8908 normam1618@gmail.com

Nereyda Fabiola Salinas. Licenciada en Geografía, UNAN MANAGUA, Departamento de Geografía https://orcid.org/0000-0002-8114-5943 nereydasalinas293@gmail.com

Lisseth Carolina Blandón Chavarría.

Master en Ciencias del agua con énfasis en calidad. UNAN MANAGUA, Departamento de Geografía.

https://orcid.org/0000-0003-4125-0332 lizzblandon@gmail.com 